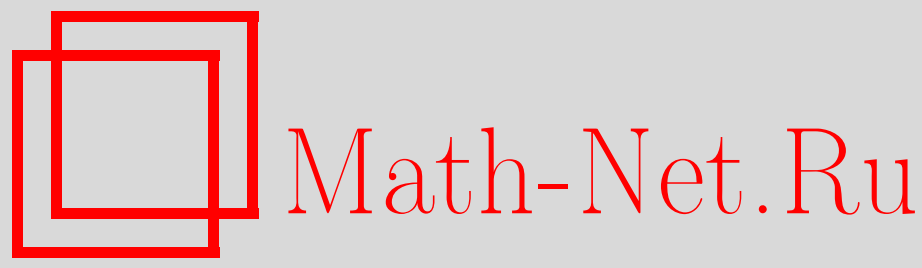

Р. К. Гупта, К. Сингла, Симметрийный анализ систем нелинейных уравнений в частных производных дробного порядка по времени с переменными коэффициентами, ТМФ, 2018, том 197, номер 3, 397-416

DOI: https://doi.org/10.4213/tmf9447

Использование Общероссийского математического портала Math-Net.Ru подразумевает, что вы прочитали и согласны с пользовательским соглашением http://www.mathnet.ru/rus/agreement

Параметры загрузки:

IP : 18.209 .158 .208

26 апреля 2023 г., 16:13:24

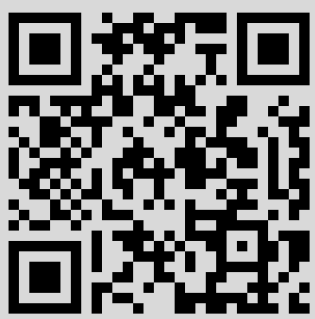




\title{
СИММЕТРИЙНЫЙ АНАЛИЗ СИСТЕМ НЕЛИНЕЙНЫХ УРАВНЕНИЙ В ЧАСТНЫХ ПРОИЗВОДНЫХ ДРОБНОГО ПОРЯДКА ПО ВРЕМЕНИ С ПЕРЕМЕННЫМИ КОЭФФИЦИЕНТАМИ
}

\begin{abstract}
С использованием метода симметрий Ли исследованы некоторые известные системы нелинейных уравнений в частных производных с дробными производными по времени и переменными коэффициентами. Получены их симметрии и редукции к системам нелинейных обыкновенных дифференциальных уравнений дробного порядка.
\end{abstract}

Ключевые слова: симметрийный анализ, системы нелинейных уравнений дробного порядка, дифференциальные уравнения в частных производных с переменными коэффициентами, операторы Эрдейи-Кобера.

DOI: https://doi.org/10.4213/tmf9447

\section{1. ВВЕДЕНИЕ}

Вначале изучение производных дробного порядка ограничивалось только математическим сообществом. Ученым потребовалось много времени, чтобы признать тот факт, что дробные производные имеют потенциал для описания природных явлений [1]-[3]. Математическое моделирование систем в терминах дробных производных приводит к дифференциальным уравнениям дробного порядка и, следовательно, к необходимости их решения [3]. Дифференциальные уравнения дробного порядка имеют большое значение из-за их широкого применения в физике, при

P. K. Гупта благодарит University Grants Commission Центрального университета Пенджаба (Бхатинда, Индия) за поддержку данного исследования в рамках Research Award Scheme (грант F.30-105/2016 (SA-II)).

${ }^{*}$ Department of Mathematics and Statistics, Central University of Punjab, Bathinda, Punjab, India. E-mail: rajeshateli@gmail.com

${ }^{\dagger}$ Department of Mathematics, School of Physical and Mathematical Sciences, Central University of Haryana, Mahendergarh, Haryana, India

${ }^{\ddagger}$ School of Mathematics, Thapar University, Patiala, Punjab, India.

E-mail: komalsingla11@gmail.com 
изучении процессов памяти, аномальной диффузии, квантовой механики, сложных сред [1]-[3].

Хорошо известным подходом к изучению дифференциальных уравнений дробного порядка является метод симметрий Ли. Первоначально он был предложен для дифференциальных уравнений целого порядка [4]-[6], а затем получил свое развитие в случае дробного порядка [7]-[10]. В недавнем исследовании [11] было предложено применять метод симметрий к системам дифференциальных уравнений в частных производных (ДУЧП), содержащих дробные производные по времени. Однако ДУЧП с переменными коэффициентами широко не обсуждались из-за сложности вычислений с участием нелокальных дробных дифференциальных операторов. С другой стороны, исследование нелинейных дифференциальных уравнений с переменными коэффициентами имеет важное значение, поскольку они широко применяются в физике, описывая, например, распространение нелинейных диспергирующих волн в неоднородных средах [12], [13]. С использованием метода симметрий были изучены только некоторые одиночные ДУЧП с переменными коэффициентами [14]. Новизна нашего исследования состоит в том, что на сегодняшний день в литературе не обсуждался симметрийный анализ нелинейных ДУЧП и систем ДУЧП высокого порядка с дробными производными по времени. Таким образом, нашей основной целью является изучение таких ДУЧП и систем ДУЧП с помощью метода симметрий Ли, где дробные производные имеют тип Римана-Лиувилля.

Структура статьи следующая. Раздел 2 посвящен инфинитезимальным симметриям и редукциям некоторых нелинейных ДУЧП дробного порядка с переменными коэффициентами, а именно дробного уравнения КдФ-Бюргерса-Курамото и дробного обобщенного уравнения КдФ седьмого порядка. В разделе 3 рассматривается применение симметрийного анализа к трем системам нелинейных уравнений дробного порядка: к одному из вариантов системы Буссинеска, системе связанных уравнений КдФ и системе Хироты-Сацумы для связанных уравнений КдФ. Раздел 4 содержит заключение.

\section{2. НЕЛИНЕЙНЫЕ ДУЧП С ДРОБНЫМИ ПРОИЗВОДНЫМИ ПО ВРЕМЕНИ И ПЕРЕМЕННЫМИ КОЭФФИЦИЕНТАМИ}

Цель данного раздела - представить применение метода симметрий Ли [9], [10] к двум нелинейным ДУЧП с дробными производными высших порядков и переменными коэффициентами. В рассматриваемых уравнениях мы полагаем, что порядок $\alpha$ производной по времени удовлетворяет неравенству $0<\alpha<1$, а коэффициенты $a(t), b(t), c(t), d(t), e(t), f(t), g(t)$ являются ненулевыми функциями от $t$. Явные симметрии Ли этих уравнений позволяют редуцировать ДУЧП к обыкновенным дифференциальным уравнениям (ОДУ) дробного порядка в терминах операторов Эрдейи-Кобера.

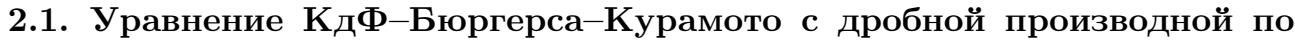
времени. Уравнение КдФ-Бюргерса-Курамото [15], [16] имеет широкий диапазон применений ко многим физическим явлениям, особенно в теории солитонов. Уравнение КдФ-Бюргерса-Курамото четвертого порядка с дробной производной по времени [15] записывается как

$$
\partial_{t}^{\alpha} u+a(t) u u_{x}+b(t) u_{x x}+c(t) u_{3 x}+u_{4 x}=0,
$$


где $u_{j x}=\partial^{j} u / \partial x^{j}$, а $\partial_{t}^{\alpha} u=\partial^{\alpha} u / \partial t^{\alpha}$ обозначает дробную производную Римана-Лиувилля [1], [3]; нижние индексы здесь и далее обозначают частные производные. Предположим, что генератор симметрии, допустимой для этого уравнения, имеет вид

$$
X=\xi(x, t, u) \partial_{x}+\tau(x, t, u) \partial_{t}+\eta(x, t, u) \partial_{u}
$$

Тогда критерий инвариантности для уравнения (1) можно записать как

$$
\begin{aligned}
{\left[\eta^{\alpha, t}+a(t)\left(\eta u_{x}+u \eta^{x}\right)\right.} & +b(t) \eta^{2 x}+c(t) \eta^{3 x}+ \\
& \left.+\tau\left(a^{\prime}(t) u u_{x}+b^{\prime}(t) u_{2 x}+c^{\prime}(t) u_{3 x}\right)+\eta^{4 x}\right]\left.\right|_{(1)}=0
\end{aligned}
$$

Подставляя инфинитезимальные операторы продолжения [8]-[10] и сравнивая коэффициенты при различных производных и степенях функции $u$, получаем следующую переопределенную систему нелинейных ДУЧП целого и дробного порядка:

$$
\begin{gathered}
\xi_{t}=\xi_{u}=0, \quad \tau_{x}=\tau_{u}=0, \quad \eta_{u u}=0, \\
\alpha \tau_{t}-4 \xi_{x}=0, \quad\left(\begin{array}{c}
\alpha \\
n
\end{array}\right) \partial_{t}^{n} \eta_{u}-\left(\begin{array}{c}
\alpha \\
n+1
\end{array}\right) \mathrm{D}_{t}^{n+1} \tau=0, \\
a(t) u\left(\alpha \tau_{t}-\xi_{x}\right)+\eta a(t)+\tau a^{\prime}(t) u+ \\
+b(t)\left(2 \eta_{x u}-\xi_{x x}\right)+c(t)\left(3 \eta_{x x u}-\xi_{x x x}\right)+\left(4 \eta_{x x u}-\xi_{x x x}\right)=0, \\
b(t)\left(\alpha \tau_{t}-2 \xi_{x}\right)+\tau b^{\prime}(t)+3 c(t)\left(\eta_{x u}-\xi_{x x}\right)+\left(6 \eta_{x x u}-4 \xi_{x x x}\right)=0, \\
c(t) u\left(\alpha \tau_{t}-3 \xi_{x}\right)+\tau c^{\prime}(t) u+\left(4 \eta_{x u}-6 \xi_{x x}\right)=0 \\
\partial_{t}^{\alpha} \eta-u \partial_{t}^{\alpha} \eta_{u}+a(t) u \eta_{x}+b(t) \eta_{x x}+c(t) u \eta_{x x x}+\eta_{4 x}=0 .
\end{gathered}
$$

Здесь $\mathrm{D}_{t}$ обозначает оператор полной производной, а штрихом обозначены производные первого порядка. Решение системы (4) с начальным условием $\left.\tau\right|_{t=0}=0$ имеет вид

$$
\xi=\frac{C_{1} x}{4}+C_{2}, \quad \tau=\frac{C_{1} t}{\alpha}, \quad \eta=C_{3} u,
$$

где $C_{1}, C_{2}, C_{3}$ - произвольные постоянные, удовлетворяющие уравнениям

$$
\begin{gathered}
a(t)\left(C_{3}+\frac{3 C_{1}}{4}\right)+\left(\frac{C_{1} t}{\alpha}\right) a^{\prime}(t)=0 \\
b(t)\left(\frac{C_{1}}{2}\right)+\left(\frac{C_{1} t}{\alpha}\right) b^{\prime}(t)=0, \quad c(t)\left(\frac{C_{1}}{4}\right)+\left(\frac{C_{1} t}{\alpha}\right) c^{\prime}(t)=0 .
\end{gathered}
$$

Таким образом, генератор симметрий первого порядка, допускающихся дробным ДУЧП (1), можно записать следующим образом:

$$
X=\left(\frac{C_{1} x}{4}+C_{2}\right) \partial_{x}+\left(\frac{C_{1} t}{\alpha}\right) \partial_{t}+\left(C_{3} u\right) \partial_{u}
$$

Отсюда получаются ассоциированные векторные поля

$$
V_{1}=\frac{x}{4} \partial_{x}+\frac{t}{\alpha} \partial_{t}, \quad V_{2}=\partial_{x}, \quad V_{3}=u \partial_{u}
$$


Поскольку любая линейная комбинация генераторов также является инфинитезимальным генератором, для любой линейной комбинации вышеупомянутых векторных полей можно получить симметрийную редукцию вида

$$
a_{1} V_{1}+a_{2} V_{2}+a_{3} V_{3} \text {. }
$$

Однако таких возможных линейных комбинаций бесконечно много, поэтому необходимо определить те из них, которые порождают неэквивалентные симметрийные редукции. Это приводит к понятию оптимальной системы [4]-[6]. Оптимальная система генераторов (8) имеет следующие компоненты: $V_{1}, V_{3}+r V_{1}$.

Следующим шагом является вычисление симметрийных редукций для двух компонент оптимального множества. Напомним, что левый дробный дифференциальный оператор Эрдейи-Кобера задается как [9], [17]

$$
\left(\mathcal{P}_{\delta}^{\zeta, \alpha} h\right)(z):=\left(\prod_{j=0}^{m-1}\left(\zeta+j-\frac{1}{\delta} z \frac{d}{d z}\right)\right)\left(\mathcal{K}_{\delta}^{\zeta+\alpha, m-\alpha} h\right)(z),
$$

где

$$
z>0, \quad \delta>0, \quad \alpha>0, \quad m=\left\{\begin{array}{cc}
{[\alpha]+1,} & \text { если } \alpha \notin \mathbb{N}, \\
\alpha, & \text { если } \alpha \in \mathbb{N},
\end{array}\right.
$$

и

$$
\left(\mathcal{K}_{\delta}^{\zeta, \alpha} h\right)(z):=\left\{\begin{array}{cc}
\frac{1}{\Gamma(\alpha)} \int_{1}^{\infty}(s-1)^{\alpha-1} s^{-(\zeta+\alpha)} h\left(z s^{1 / \delta}\right) d s, & \text { если } \alpha>0, \\
h(z), & \text { если } \alpha=0,
\end{array}\right.
$$

есть дробный интегральный оператор Эрдейи-Кобера.

Для генератора $V_{1}$, используя переменные и преобразования подобия из табл. 1 , можно провести редукцию дробного ДУЧП (1), как описано в следующей теореме.

Теорема 1. Для любого $\alpha>0$ преобразование $u=F(z)$ с $z=x t^{-\alpha / 4}$ редуиирует уравнение (1) к следующему нелинейному ОДУ дробного порядка:

$$
\left(\mathcal{P}_{4 / \alpha}^{1-\alpha, \alpha} F\right)(z)+a F F^{\prime}(z)+b F^{\prime \prime}(z)+c F^{\prime \prime \prime}(z)+F^{\prime \prime \prime \prime}(z)=0,
$$

где $\left(\mathcal{P}_{4 / \alpha}^{1-\alpha, \alpha} F\right)(z)$ - дробный дифференциалъный оператор Эрдейи-Кобера (10).

ДокАЗАТЕЛЬство. Прежде всего получим дробную производную Римана-Лиувилля функции $u=F(z)$ при $z=x t^{-\alpha / 4}$ для всех $\alpha$, удовлетворяющих неравенству $n-1<\alpha<n(n \in \mathbb{N})$ :

$$
\frac{\partial^{\alpha} u}{\partial t^{\alpha}}=\frac{\partial^{n}}{\partial t^{n}}\left[\frac{1}{\Gamma(n-\alpha)} \int_{0}^{t}(t-s)^{n-\alpha-1} F\left(x s^{-\alpha / 4}\right) d s\right] .
$$

Пусть $w=t / s$, тогда мы можем переписать последнее выражение как

$$
\begin{aligned}
\frac{\partial^{\alpha} u}{\partial t^{\alpha}} & =\frac{\partial^{n}}{\partial t^{n}}\left[\frac{t^{n-\alpha}}{\Gamma(n-\alpha)} \int_{1}^{\infty}(w-1)^{n-\alpha-1} w^{-(n-\alpha+1)} F\left(z w^{\alpha / 4}\right) d w\right]= \\
& =\frac{\partial^{n}}{\partial t^{n}}\left[t^{n-\alpha}\left(\mathcal{K}_{4 / \alpha}^{1, n-\alpha} F\right)(z)\right] .
\end{aligned}
$$


ТАБлицА 1. Симметрийные редукции дробного ДУЧП (1) к нелинейным ОДУ дробного порядка.

\begin{tabular}{|c|c|c|c|c|}
\hline $\begin{array}{c}\text { Гене- } \\
\text { раторы }\end{array}$ & Инварианты & Анзац & $\begin{array}{c}\text { Переменные } \\
\text { коэффициенты }\end{array}$ & $\begin{array}{c}\text { Редуцированные } \\
\text { дробные ОДУ }\end{array}$ \\
\hline$V_{1}$ & $\left(x t^{-\alpha / 4}, u\right)$ & $u=F\left(x t^{-\alpha / 4}\right)$ & $\begin{aligned} a(t) & =a t^{-3 \alpha / 4} \\
b(t) & =b t^{-\alpha / 2} \\
c(t) & =c t^{-\alpha / 4}\end{aligned}$ & уравнение (13) \\
\hline$V_{3}+r V_{1}$ & $\left(x t^{-\alpha / 4}, x^{-4 / r} u\right)$ & $u=x^{4 / r} F\left(x t^{-\alpha / 4}\right)$ & $\begin{array}{l}a(t)=a t^{-(1+3 r / 4) \alpha / r} \\
b(t)=b t^{-\alpha / 2} \\
c(t)=c t^{-\alpha / 4}\end{array}$ & уравнение (20) \\
\hline
\end{tabular}

Это соотношение выполнено и для $\alpha=n=1,2, \ldots$, поскольку $\left(\mathcal{K}_{\delta}^{\zeta, 0} F\right)(z)=F(z)$. Также для дифференцируемых функций $\psi(z)$, где $z=x t^{-\alpha / 4}$, должно выполняться следующее равенство:

$$
t \frac{\partial}{\partial t} \psi(z)=-\frac{\alpha}{4} z \frac{d}{d z} \psi(z) .
$$

Следовательно, можно упростить выражение (15) следующим образом:

$$
\frac{\partial^{n}}{\partial t^{n}}\left[t^{n-\alpha}\left(\mathcal{K}_{4 / \alpha}^{1, n-\alpha} F\right)(z)\right]=\frac{\partial^{n-1}}{\partial t^{n-1}}\left[t^{n-\alpha-1}\left(n-\alpha-\frac{\alpha}{4} z \frac{d}{d z}\right)\left(\mathcal{K}_{4 / \alpha}^{1, n-\alpha} F\right)(z)\right] .
$$

Повторяя данную процедуру $n-1$ раз, получаем следующий результат:

$$
\frac{\partial^{n}}{\partial t^{n}}\left[t^{n-\alpha}\left(\mathcal{K}_{4 / \alpha}^{1, n-\alpha} F\right)(z)\right]=t^{-\alpha} \prod_{j=0}^{n-1}\left(1-\alpha+j-\frac{\alpha}{4} z \frac{d}{d z}\right)\left(\mathcal{K}_{4 / \alpha}^{1, n-\alpha} F\right)(z) .
$$

Подставим это равенство в (15) и получим окончательное выражение для дробной производной по времени:

$$
\frac{\partial^{\alpha} u}{\partial t^{\alpha}}=t^{-\alpha}\left(\mathcal{P}_{4 / \alpha}^{1-\alpha, \alpha} F\right)(z), \quad \alpha>0
$$

Для генератора $V_{1}$, являющегося решением уравнений (6), переменные коэффициенты можно получить, как указано в табл. 1. Это завершает доказательство теоремы.

Для генератора $V_{3}+r V_{1}$ инвариантные решения можно найти, пользуясь табл. 1 . Далее, следуя рассуждениям теоремы 1 , проиллюстрируем редуцированное уравнение следующей теоремой.

Теорема 2. Для любого $\alpha>0$ дробное уравнение (1) можно редуцировать $к$ нелинейному ОДУ дробного порядка

$$
\begin{aligned}
\left(\mathcal{P}_{4 / \alpha}^{1-\alpha, \alpha} F\right)(z) & +A_{1}(z) F(z)+A_{2}(z) F^{2}(z)+A_{3}(z) F^{\prime}(z)+ \\
& +A_{4}(z) F F^{\prime}(z)+A_{5}(z) F^{\prime \prime}(z)+A_{6}(z) F^{\prime \prime \prime}(z)+F^{\prime \prime \prime \prime}(z)=0
\end{aligned}
$$


где $A_{i}(z)(i=1, \ldots, 6)$ задаются как

$$
\begin{aligned}
& A_{1}(z)=\frac{4 b}{r}\left(\frac{4}{r}-1\right) z^{-2}+\frac{4 c}{r}\left(\frac{4}{r}-1\right)\left(\frac{4}{r}-2\right) z^{-3}+\frac{12}{r}\left(\frac{4}{r}-1\right)\left(\frac{4}{r}-2\right)\left(\frac{4}{r}-3\right) z^{-4}, \\
& A_{2}(z)=\frac{4 a}{r} z^{4 / r-1}, \quad A_{3}(z)=\frac{8 b}{r} z^{-1}+\frac{12 c}{r}\left(\frac{4}{r}-1\right) z^{-2}+\frac{16}{r}\left(\frac{4}{r}-1\right)\left(\frac{4}{r}-1\right) z^{-3}, \\
& A_{4}(z)=a z^{4 / r}, \quad A_{5}(z)=b+\frac{12 c}{r} z^{-1}+\frac{24}{r}\left(\frac{4}{r}-1\right) z^{-2}, \quad A_{6}(z)=c+\frac{16}{r} z^{-1} .
\end{aligned}
$$

\section{2. Обощенное уравнение КдФ седьмого порядка с дробной производ-} ной по времени. Уравнения с дробной производной по времени типа уравнения КдФ возникают во многих задачах теоретической физики, например в теории длинных волн на мелкой воде и физике плазмы [18]. Рассматриваемое нами обощенное уравнение КдФ седьмого порядка с дробной производной по времени и переменными коэффициентами имеет вид [19]

$$
\begin{aligned}
\partial_{t}^{\alpha} u & +a(t) u^{3} u_{x}+b(t) u_{x}^{3}+c(t) u u_{x} u_{x x}+d(t) u^{2} u_{3 x}+ \\
& +e(t) u_{x x} u_{3 x}+f(t) u_{x} u_{4 x}+g(t) u u_{5 x}+u_{7 x}=0 .
\end{aligned}
$$

Определяющие уравнения можно получить, используя расширенные операторы симметрии в соответствующем критерии инвариантности и приравнивая коэффициенты. Решение полученной системы уравнений дает следующие ифинитезимальные величины:

$$
\xi=\frac{C_{1} x}{7}+C_{2}, \quad \tau=\frac{C_{1} t}{\alpha}, \quad \eta=C_{3} u,
$$

где $C_{1}, C_{2}, C_{3}$ - произвольные постоянные. Переменные коэффициенты должны удовлетворять следующим уравнениям:

$$
\begin{array}{cc}
a(t)\left(3 C_{3}+\frac{6 C_{1}}{7}\right)+\left(\frac{C_{1} t}{\alpha}\right) a^{\prime}(t)=0, & b(t)\left(2 C_{3}+\frac{4 C_{1}}{7}\right)+\left(\frac{C_{1} t}{\alpha}\right) b^{\prime}(t)=0, \\
c(t)\left(2 C_{3}+\frac{4 C_{1}}{7}\right)+\left(\frac{C_{1} t}{\alpha}\right) c^{\prime}(t)=0, & d(t)\left(2 C_{3}+\frac{4 C_{1}}{7}\right)+\left(\frac{C_{1} t}{\alpha}\right) d^{\prime}(t)=0, \\
e(t)\left(C_{3}+\frac{2 C_{1}}{7}\right)+\left(\frac{C_{1} t}{\alpha}\right) e^{\prime}(t)=0, & f(t)\left(C_{3}+\frac{2 C_{1}}{7}\right)+\left(\frac{C_{1} t}{\alpha}\right) f^{\prime}(t)=0, \\
g(t)\left(C_{3}+\frac{2 C_{1}}{7}\right)+\left(\frac{C_{1} t}{\alpha}\right) g^{\prime}(t)=0 .
\end{array}
$$

Соответствующие инфинитезимальные генераторы задаются как

$$
V_{1}=\frac{x}{7} \partial_{x}+\frac{t}{\alpha} \partial_{t}, \quad V_{2}=\partial_{x}, \quad V_{3}=u \partial_{u}
$$

и оптимальная система натянута на следующие генераторы симметрии:

$$
V_{1}, \quad V_{3}+r V_{1}
$$

В табл. 2 перечислены инварианты, генераторы преобразований подобия, переменные коэффициенты и пр. для генераторов (25). Симметрийные редукции для обоих генераторов симметрии обсуждаются в следующих теоремах. 
ТАБлицА 2. Симметрийные редукции дробного ДУЧП (21) к нелинейным ОДУ дробного порядка.

\begin{tabular}{|c|c|c|c|c|}
\hline $\begin{array}{c}\text { Гене- } \\
\text { раторы }\end{array}$ & Инварианты & Анзац & $\begin{array}{c}\text { Переменные } \\
\text { коэффициенты }\end{array}$ & $\begin{array}{c}\text { Редуцированные } \\
\text { дробные ОДУ }\end{array}$ \\
\hline$V_{1}$ & $\left(x t^{-\alpha / 7}, u\right)$ & $u=F\left(x t^{-\alpha / 7}\right)$ & $\begin{aligned} a(t) & =a t^{-6 \alpha / 7} \\
b(t) & =b t^{-4 \alpha / 7} \\
c(t) & =c t^{-4 \alpha / 7} \\
d(t) & =d t^{-4 \alpha / 7} \\
e(t) & =e t^{-2 \alpha / 7} \\
f(t) & =f t^{-2 \alpha / 7} \\
g(t) & =g t^{-2 \alpha / 7}\end{aligned}$ & Уравнение (26) \\
\hline$V_{3}+r V_{1}$ & $\left(x t^{-\alpha / 7}, x^{-7 / r} u\right)$ & $u=x^{7 / r} F\left(x t^{-\alpha / 7}\right)$ & $\begin{aligned} a(t) & =a t^{-(3+6 r / 7) \alpha / r}, \\
b(t) & =b t^{-(2+4 r / 7) \alpha / r}, \\
c(t) & =c t^{-(2+4 r / 7) \alpha / r}, \\
d(t) & =d t^{-(2+4 r / 7) \alpha / r}, \\
e(t) & =e t^{-(1+2 r / 7) \alpha / r}, \\
f(t) & =f t^{-(1+2 r / 7) \alpha / r}, \\
g(t) & =g t^{-(1+2 r / 7) \alpha / r}\end{aligned}$ & Уравнение (27) \\
\hline
\end{tabular}

Теорема 3. Для любого $\alpha>0$ преобразование $u=F(z)$ с $z=x t^{-\alpha / 7}$ редуцирует уравнение (21) в случае генератора $V_{1} \kappa$ следующему нелинейному ОДУ дробного порядка:

$$
\begin{aligned}
\left(\mathcal{P}_{7 / \alpha}^{1-\alpha, \alpha} F\right)(z) & +a F^{3} F^{\prime}(z)+b F^{3}(z)+c F F^{\prime} F^{\prime \prime}(z)+d F^{2} F^{\prime \prime \prime}(z)+ \\
& +e F^{\prime \prime} F^{\prime \prime \prime}(z)+f F^{\prime} F^{\prime \prime \prime \prime}(z)+g F F^{\prime \prime \prime \prime \prime}(z)+F^{\prime \prime \prime \prime \prime \prime \prime \prime ~}(z)=0,
\end{aligned}
$$

где $\left(\mathcal{P}_{7 / \alpha}^{1-\alpha, \alpha} F\right)(z)$ - дробный дифференииальный оператор Эрдейи-Кобера.

ТЕОРема 4. Для любого $\alpha>0$ ДУЧП (21) с дробной производной по времени в случае генератора $V_{3}+r V_{1}$ можно редуцировать $\kappa$ нелинейному ОДУ дробного порядка

$$
\begin{aligned}
\left(\mathcal{P}_{7 / \alpha}^{1-\alpha, \alpha} F\right. & (z)+A_{1} z^{-7} F(z)+A_{2} z^{7 / r-5} F^{2}(z)+A_{3} z^{14 / r-3} F^{3}(z)+\frac{7 a}{r} z^{21 / r-1} F^{4}(z)+ \\
& +A_{4} z^{-6} F^{\prime}(z)+A_{5} z^{7 / r-4} F F^{\prime}(z)+A_{6} z^{7 / r-2} F^{2}(z)+A_{7} z^{14 / r-1} F F^{2}(z)+ \\
& +A_{8} z^{14 / r-2} F^{2} F^{\prime}(z)+a z^{21 / r} F^{3} F^{\prime}(z)+A_{9} z^{7 / r-3} F^{2}(z)+b z^{14 / r} F^{3}(z)+ \\
& +A_{10} z^{-5} F^{\prime \prime}(z)+A_{11} z^{7 / r-3} F F^{\prime \prime}(z)+A_{12} z^{14 / r-1} F^{2} F^{\prime \prime}(z)+ \\
& +A_{13} z^{7 / r-2} F^{\prime} F^{\prime \prime}(z)+\frac{21 e}{r} z^{7 / r-1}\left(F^{\prime \prime}\right)^{2}(z)+c z^{14 / r} F F^{\prime} F^{\prime \prime}(z)+ \\
& +A_{14} z^{-4} F^{\prime \prime \prime}(z)+A_{15} z^{7 / r-2} F F^{\prime \prime \prime}(z)+d z^{14 / r} F^{2} F^{\prime \prime \prime}(z)+ \\
& +A_{16} z^{7 / r-1} F^{\prime} F^{\prime \prime \prime}(z)+e z^{7 / r} F^{\prime \prime} F^{\prime \prime \prime}(z)+A_{17} z^{-3} F^{\prime \prime \prime \prime}(z)+ \\
& +\left(\frac{7 f}{r}+g\right) z^{7 / r-1} F F^{\prime \prime \prime \prime}(z)+f z^{7 / r} F^{\prime} F^{\prime \prime \prime \prime}(z)+A_{18} z^{-2} F^{\prime \prime \prime \prime \prime}(z)+ \\
& +g z^{7 / r} F F^{\prime \prime \prime \prime \prime}(z)+\frac{49}{r} z^{-1} F^{\prime \prime \prime \prime \prime \prime \prime}(z)+F^{\prime \prime \prime \prime \prime \prime \prime \prime}(z)=0
\end{aligned}
$$


где $A_{i}(i=1, \ldots, 18)$ задаются как

$$
\begin{aligned}
& A_{1}=\frac{823543}{r^{7}}-\frac{2470629}{r^{6}}+\frac{2941225}{r^{5}}-\frac{1764735}{r^{4}}+\frac{557032}{r^{3}}-\frac{86436}{r^{2}}+\frac{5040}{r} \text {, } \\
& A_{2}=\frac{49 e}{r^{2}}\left(\frac{7}{r}-1\right)^{2}\left(\frac{7}{r}-2\right)+\frac{7 f}{r}\left(\frac{2901}{r^{4}}-\frac{2058}{r^{3}}+\frac{539}{r^{2}}-\frac{42}{r}\right)+ \\
& +g\left(\frac{16807}{r^{5}}-\frac{24010}{r^{4}}+\frac{12005}{r^{3}}-\frac{2450}{r^{2}}+\frac{168}{r}\right) z^{7 / r-5}, \\
& A_{3}=\frac{343 b}{r^{3}}+c\left(\frac{343}{r^{3}}-\frac{49}{r^{2}}\right)+d\left(\frac{343}{r^{3}}-\frac{147}{r^{2}}\right) \text {, } \\
& A_{4}=\frac{823543}{r^{6}}-\frac{1764735}{r^{5}}+\frac{1428595}{r^{4}}-\frac{540225}{r^{3}}+\frac{93982}{r^{2}}-\frac{5880}{r}, \\
& A_{5}=\frac{49 e}{r^{2}}\left(\frac{245}{r^{2}}-\frac{84}{r}+7\right)+f\left(\frac{12005}{r^{4}}-\frac{6174}{r^{3}}+\frac{931}{r^{2}}-\frac{42}{r}\right)+ \\
& +g\left(\frac{12005}{r^{4}}-\frac{10290}{r^{3}}+\frac{2695}{r^{2}}-\frac{210}{r}\right) \\
& A_{6}=\frac{294 e}{r^{2}}\left(\frac{7}{r}-1\right), \quad A_{7}=\frac{21 b}{r}+\frac{14 c}{r}, \\
& A_{8}=\frac{147 b}{r^{2}}+c\left(\frac{147}{r^{2}}-\frac{7}{r}\right)+d\left(\frac{147}{r^{2}}-\frac{21}{r}\right), \quad A_{9}=f\left(\frac{1372}{r^{3}}-\frac{588}{r^{2}}+\frac{56}{r}\right), \\
& A_{10}=\frac{352947}{r^{5}}-\frac{504210}{r^{4}}+\frac{252105}{r^{3}}-\frac{51450}{r^{2}}+\frac{3528}{r} \text {, } \\
& A_{11}=e\left(\frac{1372}{r^{3}}-\frac{294}{r^{2}}+\frac{14}{r}\right)+f\left(\frac{2058}{r^{3}}-\frac{294}{r^{2}}\right)+g\left(\frac{3430}{r^{3}}-\frac{1470}{r^{2}}+\frac{140}{r}\right), \\
& A_{12}=\frac{7 c}{r}+\frac{21 d}{r}, \quad A_{13}=e\left(\frac{441}{r^{2}}-\frac{21}{r}\right)+f\left(\frac{294}{r^{2}}-\frac{42}{r}\right) \text {, } \\
& A_{14}=\frac{84035}{r^{4}}-\frac{72030}{r^{3}}+\frac{18865}{r^{2}}-\frac{1470}{r}, \quad A_{15}=e\left(\frac{49}{r^{2}}-\frac{7}{r}\right)+\frac{7 f}{r}+g\left(\frac{490}{r^{2}}-\frac{70}{r}\right) \text {, } \\
& A_{16}=\frac{14 e}{r}+\frac{28 f}{r}, \quad A_{17}=\frac{12005}{r^{3}}-\frac{5145}{r^{2}}+\frac{490}{r}, \quad A_{18}=\frac{1029}{r^{2}}-\frac{147}{r} .
\end{aligned}
$$

\section{3. СИСТЕМА НЕЛИНЕЙНЫХ ДУЧП С ДРОБНОЙ ПРОИЗВОДНОЙ ПО ВРЕМЕНИ И ПЕРЕМЕННЫМИ КОЭФФИЦИЕНТАМИ}

В данном разделе с использованием подхода симметрий Ли исследуются три физически значимые системы нелинейных уравнений с дробной производной по времени и переменными коэффициентами. Мы полагаем, что $0<\alpha<1$ и $a(t), b(t)$, $c(t), d(t), e(t), f(t), g(t)$ - ненулевые функции от $t$. Основная идея состоит в том, чтобы сформулировать систематическую процедуру задания инфинитезимальных симметрий исследуемых уравнений и редуцировать эти системы к системам неэквивалентным между собой нелинейных ОДУ дробного порядка. 


\section{1. Вариант системы Буссинеска с дробной производной по времени.} Уравнения Буссинеска [20] играют важную роль во многих областях физики, описывая, например, колебания нелинейной струны. Они также моделируют крупномасштабные атмосферные и океанические потоки, которые отвечают за холодные фронты и струйные течения [21], [22]. Решение уравнения Буссинеска целого порядка с переменными коэффициентами было найдено с помощью метода симметрий Ли в работе [23]. Система Буссинеска с дробной производной по времени [24] и с переменными коэффициентами может быть записана в следующем виде:

$$
\begin{aligned}
& \partial_{t}^{\alpha} u+a(t) v_{x}+b(t) u u_{x}+c(t) u_{x x}=0, \\
& \partial_{t}^{\alpha} v+d(t) u v_{x}+e(t) v u_{x}+f(t) v_{x x}+g(t) u_{x x x}=0 .
\end{aligned}
$$

Группа симметрий этой системы дает следующий критерий инфинитезимальной инвариантности:

$$
\begin{aligned}
& {\left[\eta^{\alpha, t}+a(t) \phi^{x}+b(t)\left(u \eta^{x}+\eta u_{x}\right)+c(t) \eta^{x x}+\right.} \\
& \left.\quad+\tau\left(a^{\prime}(t) v_{x}+b^{\prime}(t) u u_{x}+c^{\prime}(t) u_{x x}\right)\right]\left.\right|_{(28)}=0, \\
& {\left[\phi^{\alpha, t}+d(t)\left(\eta v_{x}+u \phi^{x}\right)+e(t)\left(\phi u_{x}+v \eta^{x}\right)+f(t) \phi^{x x}+g(t) \eta^{x x x}+\right.} \\
& \left.\quad+\tau\left(d^{\prime}(t) u v_{x}+e^{\prime}(t) v u_{x}+f^{\prime}(t) v_{x x}+g^{\prime}(t) u_{x x x}\right)\right]\left.\right|_{(28)}=0 .
\end{aligned}
$$

При этом ассоциированный оператор симметрии имеет вид

$$
X=\xi(x, t, u, v) \partial_{x}+\tau(x, t, u, v) \partial_{t}+\eta(x, t, u, v) \partial_{u}+\phi(x, t, u, v) \partial_{v} .
$$

Подставляя операторы продолжения из работы [11] и сравнивая коэффициенты при различных частных и дробных производных функций $u$ и $v$, получаем определяющие уравнения:

$$
\begin{gathered}
\xi_{t}=\xi_{u}=\xi_{v}=0, \quad \tau_{x}=\tau_{u}=\tau_{v}=0, \quad \eta_{v}=\eta_{u u}=0, \quad \phi_{u}=\phi_{v v}=0, \\
\left(\begin{array}{c}
\alpha \\
n
\end{array}\right) \partial_{t}^{n} \eta_{u}-\left(\begin{array}{c}
\alpha \\
n+1
\end{array}\right) D_{t}^{n+1} \tau=0, \\
\left(\begin{array}{c}
\alpha \\
n
\end{array}\right) \partial_{t}^{n} \phi_{v}-\left(\begin{array}{c}
\alpha \\
n+1
\end{array}\right) D_{t}^{n+1} \tau=0, \\
a(t)\left(\phi_{v}-\eta_{u}+\alpha \tau_{t}-\xi_{x}\right)+\tau a^{\prime}(t)=0, \quad b(t) u\left(\alpha \tau_{t}-\xi_{x}\right)+b(t) \eta+\tau b^{\prime}(t) u=0, \\
c(t)\left(\alpha \tau_{t}-2 \xi_{x}\right)+\tau c^{\prime}(t)=0, \quad g(t)\left(\alpha \tau_{t}-3 \xi_{x}\right)+\tau g^{\prime}(t)=0, \\
d(t) u\left(\alpha \tau_{t}-\xi_{x}\right)+d(t) \eta+\tau d^{\prime}(t) u+f(t)\left(2 \phi_{x v}-\xi_{x x}\right)+g(t)\left(3 \phi_{x x v}-\xi_{x x x}\right)=0, \\
e(t) v\left(\eta_{u}-\phi_{v}+\alpha \tau_{t}-\xi_{x}\right)+e(t) \phi+\tau e^{\prime}(t) v=0, \\
f(t)\left(\alpha \tau_{t}-2 \xi_{x}\right)+\tau f^{\prime}(t)+3 g(t)\left(\phi_{x v}-\xi_{x x}\right)=0, \\
\partial_{t}^{\alpha} \eta-u \partial_{t}^{\alpha} \eta_{u}+a(t) \phi_{x}+b(t) u \eta_{x}+c(t) \eta_{x x}=0, \\
\partial_{t}^{\alpha} \phi-v \partial_{t}^{\alpha} \phi_{v}+d(t) u \phi_{x}+e(t) v \eta_{x}+f(t) \phi_{x x}+g(t) \phi_{x x x}=0 .
\end{gathered}
$$

Общее решение этой системы имеет вид

$$
\begin{gathered}
\xi=A_{1} x+A_{2}, \quad \tau=\frac{1}{c^{1 / \alpha}(t)}\left(\frac{2 A_{1}}{\alpha} \int c^{1 / \alpha}(t) d t+A_{5}\right), \\
\eta=A_{3}(t) u, \quad \phi=A_{4}(t) v
\end{gathered}
$$


где $A_{1}, A_{2}, A_{5}$ - произвольные постоянные, а на функции $A_{3}(t), A_{4}(t)$ наложены условия

$$
\tau(0)=0, \quad A_{3}^{\prime}(t)=A_{4}^{\prime}(t)=\left(\frac{\alpha-1}{2}\right) \tau_{t t},
$$

$$
\begin{array}{ll}
a(t)\left(A_{3}(t)-A_{4}(t)+\alpha \tau_{t}-A_{1}\right)+\tau a^{\prime}(t)=0, & b(t)\left(A_{3}(t)+\alpha \tau_{t}-A_{1}\right)+\tau b^{\prime}(t)=0, \\
d(t)\left(A_{3}(t)+\alpha \tau_{t}-A_{1}\right)+\tau d^{\prime}(t)=0, & e(t)\left(A_{3}(t)+\alpha \tau_{t}-A_{1}\right)+\tau e^{\prime}(t)=0, \\
f(t)\left(\alpha \tau_{t}-2 A_{1}\right)+\tau f^{\prime}(t)=0, & g(t)\left(\alpha \tau_{t}-3 A_{1}\right)+\tau g^{\prime}(t)=0 .
\end{array}
$$

Понятно, что симметрии (31) являются обобщенными в отличие от симметрий в случае целого порядка [23], поскольку система (28) дробного порядка при $\alpha=1$ совпадает с системой целого порядка [23]. В общем случае весьма сложно далее редуцировать систему (28) к системе дробных ОДУ, используя инвариантные решения, получающиеся из ассоциированных векторных полей. На данный момент в частном случае $c(t)=c=$ const эта проблема считается решенной, но поиск редуцированной системы для общего случая продолжается. В этом частном случае с использованием условия $\tau(0)=0$ получаем следующие симметрии:

$$
\xi=A_{1} x+A_{2}, \quad \tau=\frac{2 A_{1} t}{\alpha}, \quad \eta=A_{3} u, \quad \phi=A_{4} v .
$$

Постоянные $A_{i}(i=1, \ldots, 4)$ и переменные коэффициенты должны удовлетворять уравнениям

$$
\begin{array}{ll}
a(t)\left(A_{4}-A_{3}+A_{1}\right)+\tau a^{\prime}(t)=0, & b(t)\left(A_{3}+A_{1}\right)+\tau b^{\prime}(t)=0, \\
d(t)\left(A_{3}+A_{1}\right)+\tau d^{\prime}(t)=0, & e(t)\left(A_{3}+A_{1}\right)+\tau e^{\prime}(t)=0, \\
f(t)=\mathrm{const}, & g(t)\left(-A_{1}\right)+\tau g^{\prime}(t)=0 .
\end{array}
$$

Ассоциированная алгебра Ли порождается генераторами симметрии

$$
V_{1}=x \partial_{x}+\frac{2 t}{\alpha} \partial_{t}, \quad V_{2}=\partial_{x}, \quad V_{3}=u \partial_{u}, \quad V_{4}=v \partial_{v} .
$$

В соответствии с концепцией оптимальной системы [4]-[6] оптимальная система генераторов (33) имеет компоненты

$$
\Delta_{1}=V_{4}+r V_{3}+s V_{1}, \quad \Delta_{2}=V_{3}+p V_{1}, \quad \Delta_{3}=V_{1} .
$$

Для генератора $\Delta_{1}$ симметрийная редукция задается следующей теоремой.

Теорема 5. Для любого $\alpha>0$ систему Буссинеска (28) с дробной производной по времени в случае генератора $\Delta_{1}$ можно редуиировать $к$ системе нелинейных ОДУ дробного порядка

$$
\begin{aligned}
& \left(\mathcal{P}_{2 / \alpha}^{1-\alpha, \alpha} F\right)(z)+\frac{c r}{s}\left(\frac{r}{s}-1\right) z^{-2} F(z)+\frac{a}{s} z^{(1-r) / s-1} G(z)+ \\
& \quad+\frac{b r}{s} z^{r / s-1} F^{2}(z)+\frac{2 c r}{s} z^{-1} F^{\prime}(z)+a z^{(1-r) / s} G^{\prime}(z)+b z^{r / s}\left(F F^{\prime}\right)(z)+c F^{\prime \prime}(z)=0 \\
& \left(\mathcal{P}_{2 / \alpha}^{1-\alpha, \alpha} G\right)(z)+\frac{1}{s}\left(\frac{1}{s}-1\right)\left(f z+g\left(\frac{1}{s}-2\right)\right) z^{-3} G(z)+ \\
& \quad+\frac{1}{s}(d+e r) z^{r / s-1}(F G)(z)+\frac{1}{s}\left(2 f z+3 g\left(\frac{1}{s}-1\right)\right) z^{-2} G^{\prime}(z)+ \\
& \quad+z^{r / s}\left(d F G^{\prime}+e G F^{\prime}\right)(z)+\left(f z+\frac{3 g}{s}\right) z^{-1} G^{\prime \prime}(z)+g G^{\prime \prime \prime}(z)=0
\end{aligned}
$$

где $\mathcal{P}_{\delta}^{\zeta, \alpha}-$ дробный оператор Эрдейи-Кобера. 
ДокАЗАТЕЛЬство. Мы имеем следующее преобразование подобия с функциями $F, G$ для генератора $\Delta_{1}$ :

$$
z=x t^{-\alpha / 2}, \quad u=x^{r / s} F(z), \quad v=x^{1 / s} G(z) .
$$

Используя определение дробной производной Римана-Лиувилля, получаем для $\alpha$ из интервала $n-1<\alpha<n$

$$
\partial_{t}^{\alpha} u=x^{r / s} \frac{\partial^{n}}{\partial t^{n}}\left[\frac{1}{\Gamma(n-\alpha)} \int_{0}^{t}(t-s)^{n-\alpha-1} F\left(x s^{-\alpha / 2}\right) d s\right] .
$$

Если положить $t / s=\lambda$, это соотношение можно переписать как

$$
\begin{aligned}
\partial_{t}^{\alpha} u & =x^{r / s} \frac{\partial^{n}}{\partial t^{n}}\left[\frac{1}{\Gamma(n-\alpha)} \int_{1}^{\infty}(\lambda-1)^{n-\alpha-1} \lambda^{-(n-\alpha+1)} F\left(z \lambda^{\alpha / 2}\right) d \lambda\right]= \\
& =x^{r / s} \frac{\partial^{n}}{\partial t^{n}}\left[t^{n-\alpha}\left(\mathcal{K}_{2 / \alpha}^{1, n-\alpha} F\right)(z)\right] .
\end{aligned}
$$

Также, используя определение оператора Эрдейи-Кобера (12), мы видим, что результат (38) имеет место для $\alpha=n=1,2, \ldots$. Следовательно, дробная производная функции $u(x, t)$ для любого $\alpha>0$ имеет вид

$$
\begin{aligned}
\partial_{t}^{\alpha} u & =x^{r / s} t^{-\alpha} \prod_{j=0}^{n-1}\left(1-\alpha+j-\frac{\alpha}{2} \frac{d}{d z}\right)\left(\mathcal{K}_{2 / \alpha}^{1, n-\alpha} F\right)(z)= \\
& =x^{r / s} t^{-\alpha}\left(\mathcal{P}_{2 / \alpha}^{1-\alpha, \alpha} F\right)(z) .
\end{aligned}
$$

Аналогичное равенство справедливо для $v(x, t)$ : для любого $\alpha>0$

$$
\partial_{t}^{\alpha} v=x^{1 / s} t^{-\alpha}\left(\mathcal{P}_{2 / \alpha}^{1-\alpha, \alpha} G\right)(z)
$$

Это завершает доказательство теоремы.

Далее можно доказать следующие утверждения для генераторов $\Delta_{2}, \Delta_{3}$.

Теорема 6. Для любого $\alpha>0$ систему (28) в случае генератора $\Delta_{2}$ можно редуцировать к системе нелинейных ОДУ дробного порядка

$$
\begin{aligned}
& \left(\mathcal{P}_{2 / \alpha}^{1-\alpha, \alpha} F\right)(z)+\frac{c}{p}\left(\frac{1}{p}-1\right) z^{-2} F(z)+\frac{b}{p} z^{1 / p-1} F^{2}(z)+\frac{2 c}{p} z^{-1} F^{\prime}(z)+ \\
& \quad+a z^{-1 / p} G^{\prime}(z)+b z^{1 / p}\left(F F^{\prime}\right)(z)+c F^{\prime \prime}(z)=0, \\
& \left(\mathcal{P}_{2 / \alpha}^{1-\alpha, \alpha} G\right)(z)+\frac{e}{p} z^{1 / p-1}(F G)(z)+ \\
& \quad+z^{1 / p}\left(d F G^{\prime}+e G F^{\prime}\right)(z)+f G^{\prime \prime}(z)+g G^{\prime \prime \prime}(z)=0 .
\end{aligned}
$$


ТАБлица 3. Симметрийные редукции системы (28) к системе нелинейных ОДУ дробного порядка.

\begin{tabular}{|c|c|c|c|c|}
\hline $\begin{array}{c}\text { Гене- } \\
\text { раторы }\end{array}$ & Инварианты & Анзац & $\begin{array}{c}\text { Переменные } \\
\text { коэффициенты }\end{array}$ & $\begin{array}{c}\text { Редуцированные } \\
\text { системы }\end{array}$ \\
\hline$\Delta_{1}$ & $\left(x t^{-\alpha / 2}, x^{-r / s} u, x^{-1 / s} v\right)$ & $\begin{array}{l}u=x^{r / s} F(z), \\
v=x^{1 / s} G(z)\end{array}$ & $\begin{aligned} a(t) & =a t^{-(1-r+s) \alpha / 2 s,} \\
b(t) & =b t^{-(r+s) \alpha / 2 s}, \\
d(t) & =d t^{-(r+s) \alpha / 2 s}, \\
e(t) & =e t^{-(r+s) \alpha / 2 s}, \\
g(t) & =g t^{\alpha / 2}\end{aligned}$ & Система (35) \\
\hline$\Delta_{2}$ & $\left(x t^{-\alpha / 2}, x^{-1 / p} u, v\right)$ & $\begin{array}{l}u=x^{1 / p} F(z) \\
v=G(z)\end{array}$ & $\begin{aligned} a(t) & =a t^{(1-p) \alpha / 2 p} \\
b(t) & =b t^{-(1+p) \alpha / 2 p} \\
d(t) & =d t^{-(1+p) \alpha / 2 p} \\
e(t) & =e t^{-(1+p) \alpha / 2 p} \\
g(t) & =g t^{\alpha / 2}\end{aligned}$ & Система (41) \\
\hline$\Delta_{3}$ & $\left(x t^{-\alpha / 2}, u, v\right)$ & $\begin{array}{l}u=F(z), \\
v=G(z)\end{array}$ & $\begin{aligned} a(t) & =a t^{-\alpha / 2} \\
b(t) & =b t^{-\alpha / 2} \\
d(t) & =d t^{-\alpha / 2} \\
e(t) & =e t^{-\alpha / 2} \\
g(t) & =g t^{-\alpha / 2}\end{aligned}$ & Система (42) \\
\hline
\end{tabular}

Теорема 7. Для любого $\alpha>0$ систему (28) в случае генератора $\Delta_{3}$ можно редуцировать к системе нелинейных ОДУ дробного порядка

$$
\begin{aligned}
& \left(\mathcal{P}_{2 / \alpha}^{1-\alpha, \alpha} F\right)(z)+a G^{\prime}(z)+b\left(F F^{\prime}\right)(z)+c F^{\prime \prime \prime}(z)=0 \\
& \left(\mathcal{P}_{2 / \alpha}^{1-\alpha, \alpha} G\right)(z)+d\left(F G^{\prime}\right)(z)+e\left(G F^{\prime}\right)(z)+f G^{\prime \prime}(z)+g G^{\prime \prime \prime}(z)=0 .
\end{aligned}
$$

Для краткости мы опускаем детали и приводим инвариантные решения, переменные коэффициенты и пр. в табл. 3.

3.2. Система связанных уравнений КдФ дробного порядка. В уравнении КдФ представлена простейшая комбинация нелинейности и дисперсии при отсутствии диссипации. Система уравнений КдФ целого порядка, а также система связанных уравнений КдФ дробного порядка с переменными коэффициентами обсуждались многими авторами с использованием различных подходов (см., например, работы [25], [26]). Система связанных уравнений КдФ дробного порядка с переменными коэффициентами [11], [26] выглядит следующим образом:

$$
\begin{aligned}
& \partial_{t}^{\alpha} u+a(t) u u_{x}+b(t) v v_{x}+c(t) u_{x x x}=0, \\
& \partial_{t}^{\alpha} v+d(t) u v_{x}+e(t) v u_{x}+f(t) v_{x x x}=0 .
\end{aligned}
$$

Используя подход симметрий Ли [11] и решая возникающие определяющие уравнения, мы получаем симметрии в явном виде:

$$
\xi=\frac{A_{1} x}{3}+A_{2}, \quad \tau=\frac{1}{c^{1 / \alpha}(t)}\left(\frac{A_{1}}{\alpha} \int c^{1 / \alpha}(t) d t+A_{5}\right), \quad \eta=A_{3}(t) u, \quad \phi=A_{4}(t) v .
$$


Для постоянных $A_{1}, A_{2}, A_{5}$ и функций $A_{3}(t), A_{4}(t)$ должны быть выполнены следующие условия:

$$
\begin{gathered}
\tau(0)=0, \quad A_{3}^{\prime}(t)=A_{4}^{\prime}(t)=\left(\frac{\alpha-1}{2}\right) \tau_{t t}, \\
a(t)\left(\alpha \tau_{t}-\frac{A_{1}}{3}-A_{3}(t)\right)+\tau a^{\prime}(t)=0, \quad b(t)\left(\alpha \tau_{t}-\frac{A_{1}}{3}-A_{3}(t)+2 A_{4}(t)\right)+\tau b^{\prime}(t)=0, \\
d(t)\left(\alpha \tau_{t}-\frac{A_{1}}{3}+A_{3}(t)\right)+\tau d^{\prime}(t)=0, \quad e(t)\left(\alpha \tau_{t}-\frac{A_{1}}{3}+A_{3}(t)\right)+\tau e^{\prime}(t)=0, \\
f(t) v\left(\alpha \tau_{t}-\frac{A_{1}}{3}\right)+\tau f^{\prime}(t)=0 .
\end{gathered}
$$

Чтобы найти редукции системы (43) к системам нелинейных ОДУ дробного порядка, положим $c(t)=c \equiv$ const и получим симметрии в упрощенном виде

$$
\xi=\frac{A_{1} x}{3}+A_{2}, \quad \tau=\frac{A_{1} t}{\alpha}, \quad \eta=A_{3} u, \quad \phi=A_{4} v ;
$$

должны выполняться уравнения

$$
\begin{array}{ll}
a(t)\left(\frac{2}{3} A_{1}-A_{3}\right)+\tau a^{\prime}(t)=0, \quad b(t)\left(\frac{2}{3} A_{1}-A_{3}+2 A_{4}\right)+\tau b^{\prime}(t)=0, \\
d(t)\left(\frac{2}{3} A_{1}+A_{3}\right)+\tau d^{\prime}(t)=0, \quad e(t)\left(\frac{2}{3} A_{1}+A_{3}\right)+\tau e^{\prime}(t)=0, \quad f(t)=\text { const. }
\end{array}
$$

Следовательно, соответствующие векторные поля имеют вид

$$
V_{1}=\frac{x}{3} \partial_{x}+\frac{t}{\alpha} \partial_{t}, \quad V_{2}=\partial_{x}, \quad V_{3}=u \partial_{u}, \quad V_{4}=v \partial_{v} .
$$

Оптимальная система содержит следующие линейные комбинации генераторов:

$$
\Delta_{1}=V_{4}+p V_{3}+q V_{1}, \quad \Delta_{2}=V_{3}+r V_{1}, \quad \Delta_{3}=V_{1}
$$

Для них редуцированные системы задаются следующими теоремами.

Теорема 8. Для любого $\alpha>0$ систему (43) в случае генератора $\Delta_{1}$ можно редуцировать к системе нелинейных ОДУ дробного порядка

$$
\begin{aligned}
& \left(\mathcal{P}_{3 / \alpha}^{1-\alpha, \alpha} F\right)(z)+\frac{3 c p}{q}\left(\frac{3 p}{q}-1\right)\left(\frac{3 p}{q}-2\right) z^{-3} F(z)+\frac{3 a p}{q} z^{3 p / q-1} F^{2}(z)+ \\
& \quad+\frac{3 b}{q} z^{(6-3 p) / q-1} G^{2}(z)+\frac{9 c p}{q}\left(\frac{3 p}{q}-1\right) z^{-2} F^{\prime}(z)+ \\
& \quad+a z^{3 p / q}\left(F F^{\prime}\right)(z)+b z^{(6-3 p) / q}\left(G G^{\prime}\right)(z)+\frac{9 c p}{q} z^{-1} F^{\prime \prime}(z)+c F^{\prime \prime \prime}(z)=0 \\
& \left(\mathcal{P}_{3 / \alpha}^{1-\alpha, \alpha} G\right)(z)+\frac{3 f}{q}\left(\frac{3}{q}-1\right)\left(\frac{3}{q}-2\right) z^{-3} G(z)+\frac{3}{q}(e p+d) z^{3 p / q-1}(F G)(z)+ \\
& \quad+z^{3 p / q}\left(d F G^{\prime}+e G F^{\prime}\right)(z)+\frac{9 f}{q}\left(\frac{3}{q}-1\right) z^{-2} G^{\prime}(z)+\frac{9 f}{q} z^{-1} G^{\prime \prime}(z)+f G^{\prime \prime \prime}(z)=0
\end{aligned}
$$

с дифберенциальным оператором Эрдейи-Кобера $\left(\mathcal{P}_{\delta}^{\zeta, \alpha}\right)$, заданнъм в (10). 
ТАБлицА 4. Симметрийные редукции системы (43) к системам нелинейных ОДУ дробного порядка

\begin{tabular}{|c|c|c|c|c|}
\hline $\begin{array}{c}\text { Гене- } \\
\text { раторы }\end{array}$ & Инварианты & Анзац & $\begin{array}{c}\text { Переменные } \\
\text { коэффициенты }\end{array}$ & $\begin{array}{c}\text { Редуцированные } \\
\text { системы }\end{array}$ \\
\hline$\Delta_{1}$ & $\left(x t^{-\alpha / 3}, x^{-3 p / q} u, x^{-3 / q} v\right)$ & $\begin{array}{l}u=x^{3 p / q} F(z), \\
v=x^{3 / q} G(z)\end{array}$ & $\begin{array}{l}a(t)=a t^{-(3 p+2 q) \alpha / 3 q}, \\
b(t)=b t^{-(6-3 p+2 q) \alpha / 3 q}, \\
d(t)=d t^{-(3 p+2 q) \alpha / 3 q}, \\
e(t)=e t^{-(3 p+2 q) \alpha / 3 q}\end{array}$ & Система (47) \\
\hline$\Delta_{2}$ & $\left(x t^{-\alpha / 3}, x^{-3 / r} u, v\right)$ & $\begin{array}{l}u=x^{3 / r} F(z), \\
v=G(z)\end{array}$ & $\begin{array}{l}a(t)=a t^{(3+2 r) \alpha / 3 r} \\
b(t)=b t^{-(-3+2 r) \alpha / 3 r} \\
d(t)=d t^{-(3+2 r) \alpha / 3 r} \\
e(t)=e t^{-(3+2 r) \alpha / 3 r}\end{array}$ & Система (48) \\
\hline$\Delta_{3}$ & $\left(x t^{-\alpha / 3}, u, v\right)$ & $\begin{array}{l}u=F(z), \\
v=G(z)\end{array}$ & $\begin{aligned} a(t) & =a t^{-2 \alpha / 3} \\
b(t) & =b t^{-2 \alpha / 3} \\
d(t) & =d t^{-2 \alpha / 3} \\
e(t) & =e t^{-2 \alpha / 3}\end{aligned}$ & Система (49) \\
\hline
\end{tabular}

Теорема 9. Для любого $\alpha>0$ систему (43) в случае генератора $\Delta_{2}$ можно редуцировать к системе нелинейных ОДУ дробного порядка

$$
\begin{aligned}
& \left(\mathcal{P}_{3 / \alpha}^{1-\alpha, \alpha} F\right)(z)+\frac{3 c}{r}\left(\frac{3}{r}-1\right)\left(\frac{3}{r}-2\right) z^{-3} F(z)+\frac{3 a}{r} z^{3 / r-1} F^{2}(z)+\frac{9 c}{r}\left(\frac{3}{r}-1\right) z^{-2} F^{\prime}(z)+ \\
& \quad+a z^{3 / r}\left(F F^{\prime}\right)(z)+b z^{-3 / r}\left(G G^{\prime}\right)(z)+\frac{9 c}{r} z^{-1} F^{\prime \prime}(z)+c F^{\prime \prime \prime}(z)=0 \\
& \left(\mathcal{P}_{3 / \alpha}^{1-\alpha, \alpha} G\right)(z)+\frac{3 e}{r} z^{3 / r-1}(F G)(z)+z^{3 / r}\left(d F G^{\prime}+e G F^{\prime}\right)(z)+f G^{\prime \prime \prime}(z)=0
\end{aligned}
$$

Теорема 10. Для любого $\alpha>0$ систему (43) в случае генератора $\Delta_{3}$ можно редуцировать к системе нелинейных ОДУ дробного порядка

$$
\begin{aligned}
& \left(\mathcal{P}_{3 / \alpha}^{1-\alpha, \alpha} F\right)(z)+a\left(F F^{\prime}\right)(z)+b\left(G G^{\prime}\right)(z)+c F^{\prime \prime \prime}(z)=0 \\
& \left(\mathcal{P}_{3 / \alpha}^{1-\alpha, \alpha} G\right)(z)+d\left(F G^{\prime}\right)(z)+e\left(G F^{\prime}\right)(z)+f G^{\prime \prime \prime}(z)=0 .
\end{aligned}
$$

Инварианты, переменные коэффициенты и симметрийные редукции перечислены в табл. 4.

3.3. Система Хироты-Сацумы связанных уравнений КдФ с дробной производной по времени. Система Хироты-Сацумы связанных уравнений КдФ имеет обширные приложения в нелинейных явлениях и изучалась многими авторами (см., например, работы [11], [27], [28]). В работе [28] данная система целого порядка с переменными коэффициентами изучалась с использованием метода групп Ли. Рассматриваемая в настоящей работе система Хироты-Сацумы связанных уравнений 
КдФ с дробной производной по времени и переменными коэффициентами записывается следующим образом:

$$
\begin{aligned}
& \partial_{t}^{\alpha} u+a(t) u u_{x}+b(t)\left(v w_{x}+w v_{x}\right)+c(t) u_{x x x}=0, \\
& \partial_{t}^{\alpha} v+d(t) u v_{x}+e(t) v_{x x x}=0, \\
& \partial_{t}^{\alpha} w+f(t) u w_{x}+g(t) w_{x x x}=0 .
\end{aligned}
$$

Пусть допустимые для данной системы генераторы симметрии имеют вид

$$
\begin{aligned}
X= & \xi(x, t, u, v, w) \partial_{x}+\tau(x, t, u, v, w) \partial_{t}+ \\
& +\eta(x, t, u, v, w) \partial_{u}+\phi(x, t, u, v, w) \partial_{v}+\psi(x, t, u, v, w) \partial_{w}
\end{aligned}
$$

Тогда критерий инвариантности записывается как

$$
\begin{aligned}
& {\left[\eta^{\alpha, t}+a\left(t\left(\eta u_{x}+u \eta^{x}\right)+b(t)\left(\phi w_{x}+v \psi^{x}+\psi v_{x}+w \phi^{x}\right)+\right.\right.} \\
& \left.\quad+\tau\left(a^{\prime}(t) u u_{x}+b^{\prime}(t)\left(v w_{x}+w v_{x}\right)\right)+\tau c^{\prime}(t) u_{x x x}+c(t) \eta^{x x x}\right]\left.\right|_{(50)}=0 \\
& {\left.\left[\phi^{\alpha, t}+d(t)\left(\eta v_{x}+u \phi^{x}\right)+\tau\left(d^{\prime}(t) u v_{x}+e^{\prime}(t) v_{x x x}\right)+e(t) \phi^{x x x}\right]\right|_{(50)}=0} \\
& {\left.\left[\psi^{\alpha, t}+f(t)\left(\eta w_{x}+u \psi^{x}\right)+\tau\left(f^{\prime}(t) u w_{x}+g^{\prime}(t) w_{x x x}\right)+g(t) \psi^{x x x}\right]\right|_{(50)}=0 .}
\end{aligned}
$$

С помощью операторов продолжения [11] мы получаем переопределенную систему ДУЧ целого и дробного порядка в указанной группе инфинитезимальных величин $\xi$, $\tau, \eta, \phi, \psi$. Решение уравнений дает симметрии Ли

$$
\begin{gathered}
\xi=A_{1} x+A_{2}, \quad \tau=\frac{1}{c^{1 / \alpha}(t)}\left(\frac{3 A_{1}}{\alpha} \int c^{1 / \alpha}(t) d t+A_{6}\right), \quad \eta=A_{3}(t) u \\
\phi=A_{4}(t) v, \quad \psi=A_{5}(t) w
\end{gathered}
$$

где $A_{1}, A_{2}, A_{6}$ - произвольные постоянные, а $A_{3}(t), A_{4}(t), A_{5}(t)$ - произвольные функции от $t$, удовлетворяющие следующим условиям:

$$
\begin{gathered}
\tau(0)=0, \quad A_{3}^{\prime}(t)=A_{4}^{\prime}(t)=A_{5}^{\prime}(t)=\left(\frac{\alpha-1}{2}\right) \tau_{t t}, \\
a(t)\left(\alpha \tau_{t}-A_{1}+A_{3}(t)\right)+\tau a^{\prime}(t)=0, \\
b(t)\left(\alpha \tau_{t}-A_{1}-A_{3}(t)+A_{4}(t)+A_{5}(t)\right)+\tau b^{\prime}(t)=0, \\
d(t)\left(\alpha \tau_{t}-A_{1}+A_{3}(t)\right)+\tau d^{\prime}(t)=0, \quad e(t)=K_{1} c(t), \\
f(t)\left(\alpha \tau_{t}-A_{1}+A_{3}(t)\right)+\tau f^{\prime}(t)=0, \quad g(t)=K_{2} c(t),
\end{gathered}
$$

в которых $K_{1}, K_{2}$ - произвольные постоянные. Далее рассмотрим частный случай $c(t)=c \equiv$ const, облегчающий расчет симметрийных редукций. В этом случае симметрии упрощаются и принимают вид

$$
\xi=A_{1} x+A_{2}, \quad \tau=\frac{3 t}{\alpha} A_{1}, \quad \eta=A_{3} u, \quad \phi=A_{4} v, \quad \psi=A_{5} w .
$$


Для коэффициентов $A_{i}(i=1, \ldots, 5)$ должны выполняться следующие уравнения:

$$
\begin{gathered}
a(t)\left(2 A_{1}+A_{3}\right)+\left(\frac{3 t}{\alpha} A_{1}\right) a^{\prime}(t)=0 \\
b(t)\left(2 A_{1}-A_{3}+A_{4}+A_{5}\right)+\left(\frac{3 t}{\alpha} A_{1}\right) b^{\prime}(t)=0 \\
d(t)\left(2 A_{1}+A_{3}\right)+\left(\frac{3 t}{\alpha} A_{1}\right) d^{\prime}(t)=0, \quad e(t)=\text { const }, \\
f(t)\left(2 A_{1}+A_{3}\right)+\left(\frac{3 t}{\alpha} A_{1}\right) f^{\prime}(t)=0, \quad g(t)=\text { const. }
\end{gathered}
$$

Соответствующие генераторы симметрии записываются как

$$
V_{1}=x \partial_{x}+\frac{3 t}{\alpha} \partial_{t}, \quad V_{2}=\partial_{x}, \quad V_{3}=u \partial_{u}, \quad V_{4}=v \partial_{v}, \quad V_{5}=w \partial_{w}
$$

Расчет показывает, что оптимальная система содержит компоненты

$$
\Delta_{1}=V_{5}+k V_{4}+l V_{3}+m V_{1}, \quad \Delta_{2}=V_{4}+r V_{3}+s V_{1}, \quad \Delta_{3}=V_{3}+\beta V_{1}, \quad \Delta_{4}=V_{1},
$$

которые приводят к редуцированным системам нелинейных уравнений дробного порядка, описанным в следующих теоремах.

Теорема 11. Для любого $\alpha>0$ систему (50) в случае генератора $\Delta_{1}$ можно редуцировать к системе нелинейных ОДУ дробного порядка

$$
\begin{aligned}
& \left(\mathcal{P}_{3 / \alpha}^{1-\alpha, \alpha} F\right)(z)+\frac{3 c l}{m}\left(\frac{l}{m}-1\right)\left(\frac{l}{m}-2\right) z^{-3} F(z)+\frac{a l}{m} z^{l / m-1} F^{2}(z)+ \\
& \quad+a z^{l / m}\left(F F^{\prime}\right)(z)+\frac{3 c l}{m}\left(\frac{l}{m}-1\right) z^{-2} F^{\prime}(z)+b z^{(1+k-l) / m-1}(1+k)(H G)(z)+ \\
& \quad+b z^{(1+k-l) / m}\left(G H^{\prime}+H G^{\prime}\right)(z)+\frac{3 c l}{m} z^{-1} F^{\prime \prime}(z)+c F^{\prime \prime \prime}(z)=0 \\
& \left(\mathcal{P}_{3 / \alpha}^{1-\alpha, \alpha} G\right)(z)+\frac{d k}{m} z^{l / m-1}(F G)(z)+\frac{3 e k}{m}\left(\frac{k}{m}-1\right)\left(\frac{k}{m}-2\right) z^{-3} G(z)+ \\
& \quad+d z^{l / m}\left(F G^{\prime}\right)(z)+\frac{3 e k}{m}\left(\frac{k}{m}-1\right) z^{-2} G^{\prime}(z)+\frac{3 e k}{m} z^{-1} G^{\prime \prime}(z)+e G^{\prime \prime \prime}(z)=0 \\
& \left(\mathcal{P}_{3 / \alpha}^{1-\alpha, \alpha} H\right)(z)+f z^{l / m-1}(F H)(z)+\frac{3 g}{m}\left(\frac{1}{m}-1\right)\left(\frac{1}{m}-2\right) z^{-3} H(z)+ \\
& \quad+f z^{l / m}\left(F H^{\prime}\right)(z)+\frac{3 g}{m}\left(\frac{1}{m}-1\right) z^{-2} H^{\prime}(z)+\frac{3 g}{m} z^{-1} H^{\prime \prime}(z)+g H^{\prime \prime \prime}(z)=0
\end{aligned}
$$

где $\left(\mathcal{P}_{\delta}^{\zeta, \alpha}\right)-$-ифференциальный оператор Эрдейи-Кобера.

ДокАзАтЕльство. Используя определение Римана-Лиувилля для дробной производной, получаем, что должно быть выполнено следующее равенство:

$$
\partial_{t}^{\alpha} u=\frac{\partial^{n}}{\partial t^{n}}\left[\frac{1}{\Gamma(n-\alpha)} \int_{0}^{t}(t-s)^{n-\alpha-1} x^{l / m} F\left(x s^{-\alpha / 3}\right) d s\right] .
$$


Положив $\rho=t / s$, для любого $\alpha>0$ перепишем это выражение как

$$
\begin{aligned}
\partial_{t}^{\alpha} u & =x^{l / m} \frac{\partial^{n}}{\partial t^{n}}\left[t^{n-\alpha}\left(\mathcal{K}_{3 / \alpha}^{1, n-\alpha} F\right)(z)\right]= \\
& =x^{l / m} t^{-\alpha} \prod_{j=0}^{n-1}\left(1-\alpha+j-\frac{\alpha}{3} \frac{d}{d z}\right)\left(\mathcal{K}_{3 / \alpha}^{1, n-\alpha} F\right)(z) .
\end{aligned}
$$

Следовательно, для любого $\alpha>0$ производную $\partial_{t}^{\alpha} u$ можно переписать в терминах дробного оператора дифференцирования Эрдейи-Кобера:

$$
\partial_{t}^{\alpha} u=x^{l / m} t^{-\alpha}\left(\mathcal{P}_{3 / \alpha}^{1-\alpha, \alpha} F\right)(z) .
$$

Аналогично для любого $\alpha>0$ получаются следующие выражения:

$$
\partial_{t}^{\alpha} v=x^{k / m} t^{-\alpha}\left(\mathcal{P}_{3 / \alpha}^{1-\alpha, \alpha} G\right)(z), \quad \partial_{t}^{\alpha} w=x^{1 / m} t^{-\alpha}\left(\mathcal{P}_{3 / \alpha}^{1-\alpha, \alpha} H\right)(z) .
$$

Отсюда, используя переменные коэффициенты из табл. 5, мы получаем утверждение теоремы.

Таким же способом выводятся симметрийные редукции для остальных генераторов $\Delta_{i}, i=2,3,4$.

Теорема 12. Для любого $\alpha>0$ систему (50) в случае генератора $\Delta_{2}$ можно редуиировать к системе нелинейных ОДУ дробного порядка

$$
\begin{aligned}
& \left(\mathcal{P}_{3 / \alpha}^{1-\alpha, \alpha} F\right)(z)+\frac{3 c r}{s}\left(\frac{r}{s}-1\right)\left(\frac{r}{s}-2\right) z^{-3} F(z)+\frac{a r}{s} z^{r / s-1} F^{2}(z)+ \\
& \quad+\frac{b}{s} z^{(1-r-s) / s}(H G)(z)+\frac{3 c r}{s}\left(\frac{r}{s}-1\right) z^{-2} F^{\prime}(z)+a z^{r / s}\left(F F^{\prime}\right)(z)+ \\
& \quad+b z^{(1-r) / s}\left(G H^{\prime}+H G^{\prime}\right)(z)+\frac{3 c r}{s} z^{-1} F^{\prime \prime}(z)+c F^{\prime \prime \prime}(z)=0 \\
& \left(\mathcal{P}_{3 / \alpha}^{1-\alpha, \alpha} G\right)(z)+\frac{3 e}{s}\left(\frac{1}{s}-1\right)\left(\frac{1}{s}-2\right) z^{-3} G(z)+\frac{d}{s} z^{r / s-1}(F G)(z)+ \\
& \quad+\frac{3 e}{s}\left(\frac{1}{s}-1\right) z^{-2} G^{\prime}(z)+d z^{r / s}\left(F G^{\prime}\right)(z)+\frac{3 e}{s} z^{-1} G^{\prime \prime}(z)+e G^{\prime \prime \prime}(z)=0 \\
& \left(\mathcal{P}_{3 / \alpha}^{1-\alpha, \alpha} H\right)(z)+f z^{r / s}\left(F H^{\prime}\right)(z)+g H^{\prime \prime \prime}(z)=0
\end{aligned}
$$

Теорема 13. Для любого $\alpha>0$ систему (50) в случае генератора $\Delta_{3}$ можно редуцировать к системе нелинейных ОДУ дробного порядка

$$
\begin{aligned}
& \left(\mathcal{P}_{3 / \alpha}^{1-\alpha, \alpha} F\right)(z)+\frac{3 c}{\beta}\left(\frac{1}{\beta}-1\right)\left(\frac{1}{\beta}-2\right) z^{-3} F(z)+ \\
& \quad+\frac{a}{\beta} z^{1 / \beta-1} F^{2}(z)+\frac{3 c}{\beta}\left(\frac{1}{\beta}-1\right) z^{-2} F^{\prime}(z)+a z^{1 / \beta}\left(F F^{\prime}\right)(z)+ \\
& \quad+b z^{-1 / \beta}\left(G H^{\prime}+H G^{\prime}\right)(z)+\frac{3 c}{\beta} z^{-1} F^{\prime \prime}(z)+c F^{\prime \prime \prime}(z)=0 \\
& \left(\mathcal{P}_{3 / \alpha}^{1-\alpha, \alpha} G\right)(z)+d z^{1 / \beta}\left(F G^{\prime}\right)(z)+e G^{\prime \prime \prime}(z)=0 \\
& \left(\mathcal{P}_{3 / \alpha}^{1-\alpha, \alpha} H\right)(z)+f z^{1 / \beta}\left(F H^{\prime}\right)(z)+g H^{\prime \prime \prime}(z)=0
\end{aligned}
$$


ТАБЛИцА 5. Симметрийные редукции системы (50) к системам нелинейных ОДУ дробного порядка

\begin{tabular}{|c|c|c|c|c|}
\hline $\begin{array}{c}\text { Гене- } \\
\text { раторы }\end{array}$ & Инварианты & Анзац & $\begin{array}{c}\text { Переменные } \\
\text { коэффициенты }\end{array}$ & $\begin{array}{c}\text { Редуцированные } \\
\text { системы }\end{array}$ \\
\hline$\Delta_{1}$ & $\begin{array}{l}\left(x t^{-\alpha / 3}, x^{-l / m} u\right. \\
\left.x^{-k / m} v, x^{-1 / m} w\right)\end{array}$ & $\begin{aligned} u & =x^{l / m} F(z), \\
v & =x^{k / m} G(z), \\
w & =x^{1 / m} H(z)\end{aligned}$ & $\begin{array}{l}a(t)=a t^{-(2 m+l) \alpha / 3 m}, \\
b(t)=b t^{-(1+2 m-l+k) \alpha / 3 m}, \\
d(t)=d t^{-(2 m+l) \alpha / 3 m}, \\
f(t)=f t^{-(2 m+l) \alpha / 3 m}\end{array}$ & Система (56) \\
\hline$\Delta_{2}$ & $\begin{array}{r}\left(x t^{-\alpha / 3}, x^{-r / s} u\right. \\
\left.x^{-1 / s} v, w\right)\end{array}$ & $\begin{aligned} u & =x^{r / s} F(z) \\
v & =x^{1 / s} G(z) \\
w & =H(z)\end{aligned}$ & $\begin{array}{l}a(t)=a t^{-(2 s+r) \alpha / 3 s} \\
b(t)=b t^{-(1+2 s-r) \alpha / 3 s} \\
d(t)=d t^{-(2 s+r) \alpha / 3 s} \\
f(t)=f t^{-(2 s+r) \alpha / 3 s}\end{array}$ & Система (61) \\
\hline$\Delta_{3}$ & $\left(x t^{-\alpha / 3}, x^{-1 / \beta} u, v, w\right)$ & $\begin{aligned} u & =x^{1 / \beta} F(z) \\
v & =G(z) \\
w & =H(z)\end{aligned}$ & $\begin{aligned} a(t) & =a t^{-(2 \beta+1) \alpha / 3 \beta} \\
b(t) & =b t^{-(2 \beta-1) \alpha / 3 \beta} \\
d(t) & =d t^{-(2 \beta+1) \alpha / 3 \beta} \\
f(t) & =f t^{-(2 \beta+1) \alpha / 3 \beta}\end{aligned}$ & Система (62) \\
\hline$\Delta_{4}$ & $\left(x t^{-\alpha / 3}, u, v, w\right)$ & $\begin{aligned} u & =F(z), \\
v & =G(z) \\
w & =H(z)\end{aligned}$ & $\begin{aligned} a(t) & =a t^{-2 \alpha / 3} \\
b(t) & =b t^{-2 \alpha / 3} \\
d(t) & =d t^{-2 \alpha / 3} \\
f(t) & =f t^{-2 \alpha / 3}\end{aligned}$ & Система (63) \\
\hline
\end{tabular}

Теорема 14. Для любого $\alpha>0$ в случае генератора $\Delta_{4}$ система (50) редуцируется к системе нелинейных ОДУ дробного порядка

$$
\begin{aligned}
& \left(\mathcal{P}_{3 / \alpha}^{1-\alpha, \alpha} F\right)(z)+a\left(F F^{\prime}\right)(z)+b\left(G H^{\prime}+H G^{\prime}\right)(z)+c F^{\prime \prime \prime}(z)=0, \\
& \left(\mathcal{P}_{3 / \alpha}^{1-\alpha, \alpha} G\right)(z)+d\left(F G^{\prime}\right)(z)+e G^{\prime \prime \prime}(z)=0, \\
& \left(\mathcal{P}_{3 / \alpha}^{1-\alpha, \alpha} H\right)(z)+f\left(F H^{\prime}\right)(z)+g H^{\prime \prime \prime}(z)=0 .
\end{aligned}
$$

В табл. 5 приведен краткий список инвариантных решений и симметрийных редукций для системы Хироты-Сацумы (50) связанных уравнений КдФ с дробной производной по времени.

\section{4. ЗАКЛЮЧЕНИЕ}

В представленной работе мы показали эффективность метода симметрий Ли, успешно исследовав два нелинейных ДУЧП высокого порядка с дробными производными по времени и три системы дробных нелинейных ДУЧП с переменными коэффициентами. Мы обсудили полную групповую классификацию уравнения КдФ-Бюргерса-Курамото четвертого порядка и обобщенного уравнения КдФ седьмого порядка, позволившую получить их редукции к нелинейным ОДУ в терминах 
операторов Эрдейи-Кобера. Также мы рассмотрели симметрийный анализ трех систем дробных нелинейных ДУЧП с переменными коэффициентами. В сравнении с соответствующими системами целого порядка мы получили обобщенные результаты для систем уравнений с дробными производными по времени. Полученные инфинитезимальные операторы сгруппированы в классы неэквивалентных генераторов оптимальной системы. Каждый генератор оптимальных систем был использован для получения групповых инвариантных решений, приводящих к редуцированным системам нелинейных ОДУ.

Нам представляется очень важным дополнительно распространить результаты и методы настоящей работы на системы уравнений с пространственными и временными дробными производными и с переменными коэффициентами.

\section{Список литературы}

[1] A. A. Kilbas, H. M. Srivastava, J. J. Trujillo, Theory and Applications of Fractional Differential Equations, North-Holland Mathematics Studies, 204, Elsevier, Amsterdam, 2006.

[2] С. Г. Самко, А. А. Килбас, О. И. Маричев, Интегралы и производные дробного порядка и некоторые их приложения, Наука и техника, Минск, 1987.

[3] I. Podlubny, An Introduction to Fractional Derivatives, Fractional Differential Equations, to Methods of Their Solution and Some of Their Applications, Fractional Differential Equations, 198, Academic Press, San Diego, CA, 1999.

[4] G. W. Bluman, S. C. Anco, Symmetry and Integration Methods for Differential Equations, Springer, New York, 2002.

[5] П. Олвер, Приложения групп Ли к дифференциальным уравнениям, Мир, М., 1989.

[6] Л. В. Овсянников, Групповой анализ дифференииальных уравнений, М., Наука, 1978.

[7] E. Buckwar, Y. Luchko, "Invariance of a partial differential equation of fractional order under the Lie group of scaling transformations", J. Math. Anal. Appl., 227:1 (1998), 81-97.

[8] Р. К. Газизов, А. А. Касаткин, С. Ю. Лукащук, "Непрерывные группы преобразований дифференциальных уравнений дробного порядка", Вестн. УГАТУ, 9:32(21) (2007), $125-135$.

[9] G. W. Wang, X. Q. Liu, Y.Y. Zhang, "Lie symmetry analysis to the time fractional generalized fifth-order KdV equation", Commun. Nonlinear Sci. Numer. Simul., 18:9 (2013), 2321-2326.

[10] Q. Huang, R. Zhdanov, "Symmetries and exact solutions of the time fractional Harry-Dym equation with Riemann-Liouville derivative", Phys. A, 409 (2014), 110-118.

[11] K. Singla, R. K. Gupta, "On invariant analysis of some time fractional nonlinear systems of partial differential equations. I", J. Math. Phys., 57:10 (2016), 101504, 14 pp.

[12] N. A. Lai, Z. Yi, "Global existence of critical nonlinear wave equation with time dependent variable coefficients", Commun. Partial Diff. Equations, 37:11 (2012), 1913-1939.

[13] T. Su, H.-H. Dai, X. Geng, "On the application of a generalized dressing method to the integration of variable-coefficient coupled Hirota equations", J. Math. Phys., 50:11 (2009), 113507, 12 pp.

[14] S. Yu. Lukashchuk, A. V. Makunin, "Group classification of nonlinear time-fractional diffusion equation with a source term", Appl. Math. Comput., 257 (2015), 335-343.

[15] Z. Fu, S. Liu, S. Liu, "New exact solution to the KdV-Burgers-Kuramato equation", Chaos, Solitons and Fractals, 23:2 (2005), 609-616.

[16] M. A. Akinlar, A. Secer, M. Bayram, "Numerical solution of fractional Benney equation", Appl. Math. Inf. Sci., 8:4 (2014), 1633-1637.

[17] V.S. Kiryakova, Generalized Fractional Calculus and Applications, Pitman Research Notes in Mathematics Series, 301, Longman Scientific \& Technical, Harlow, 1994. 
[18] S. A. El-Wakil, E. M. Abulwafa, E. K. El-Shewy, A. A. Mahmoud, "Time-fractional KdV equation for plasma of two different temperature electrons and stationary ion", Phys. Plasmas, 18:9 (2011), 092116, 7 pp.

[19] Y. Pomeau, A. Ramani, B. Grammaticos, "Structural stability of the Korteweg-de Vries solitons under a singular perturbation", Phys. D, 31:1 (1988), 127-134.

[20] J. Boussinesq, "Théorie de l'intumescence liquide appelée onde solitaire ou de translation, se propageant dans un canal rectangulaire", C. R. Acad. Sci. Paris, 72 (1871), 755-778.

[21] А. Гилл, Динамика атмосферы и океана, Мир, М., 1986.

[22] A. J. Majda, Introduction to PDEs and Waves for the Atmosphere and Ocean, Courant Lecture Notes in Mathematics, 9, AMS, Providence, RI, 2003.

[23] K. Singh, R. K. Gupta, "Exact solutions of a variant Boussinesq system", Internat. J. Eng. Sci., 44:18-19 (2006), 1256-1268.

[24] M.S. Mohamed, K. A. Gepreel, "Numerical solutions for the time fractional variant Bussinesq equation by homotopy analysis method", Sci. Res. Essays, 8:44 (2013), 2163-2170.

[25] H. M. Jaradat, "Dynamic behavior of traveling wave solutions for a class for the time-space coupled fractional KdV system with time-dependent coefficients", Ital. J. Pure Appl. Math., 36 (2016), 945-958.

[26] K. Singh, R. K. Gupta, "On symmetries and invariant solutions of a coupled KdV system with variable coefficients", Internat. J. Math. Math. Sci., 2005:23 (2005), 3711-3725.

[27] H. Zhang, "New exact solutions for two generalized Hirota-Satsuma coupled KdV systems", Commun. Nonlinear Sci. Numer. Simul., 12:7 (2007), 1120-1127.

[28] K. Singh, R. K. Gupta, "Lie symmetries and exact solutions of a new generalized HirotaSatsuma coupled KdV system with variable coefficients", Internat. J. Eng. Sci., 44:3-4 (2006), 241-255.

Поступила в редакцию 14.08.2017, после доработки 15.02.2018 